\title{
EBraISMO E PSICANALISI \\ UNO SCRITTO SUL GHETTO DI TRIESTE DI UMBERTO SABA
}

\section{Andrea Lombardi*}

Resumo: A figura do poeta triestino Umberto Saba é vista à luz de sua dupla ligação: a origem judaica (que motiva a escolha do pseudônimo hebraico) e seu vínculo com a psicanálise, que marca sua biografia e constitui uma natural chave de leitura de sua produção poética.

Palavras-chave:Umberto Saba; poesia italiana contemporânea; literatura italiana; psicanálise.

$\grave{\mathrm{E}}$

certamente un paradosso che Umberto Saba (Trieste 1883. Gorizia 1957), tra gli scrittori e poeti italiani quello che più apertamente ha proclamato la propria origine ebraica, sia ebreo solo da parte di madre, il che, comunque, gli garantisce l'ascendenza ebraica secondo il Talmud. Umberto Poli ha acquisito il cognome ebraico Saba (che in ebraico significa pane) solo successivamente, come pseudonimo nella sua vita letteraria, abbandonando polemicamente il cognome del padre. $\grave{E}$ possibile che il testo che pubblichiamo ("Il ghetto di Trieste nel 1860"), uno scritto dell'inizio del secolo, al di la di un'ironia facilmente individuabile sul folklore ebraico-mediterraneo nasconda elementi rimossi rispetto alla consistenza di un legame paterno che il poeta si è preoccupato esplicitamente di rigettare. Si veda questa poesia enfaticamente autobiografica (Autobiografia n. 3):

* Professor de Literatura Italiana junto ao Departamento de Letras Modernas da FFLCH USP. 
LOMBARDI, Andrea. Ebraismo e psicanalisi: uno scritto sul ghetto di Trieste di Umberto Saba.

"Mio padre è stato per me 'l'assassino',/ fino ai vent'anni che l'ho conosciuto./ [...] Aveva in volto il mio sguardo azzurrino,/ un sorriso, in miseria, dolce e astuto./ Andò sempre pel mondo pellegrino; - più d'una donna l'ha amato e pasciuto/ Egli era gaio e leggero; mia madre/ tutti sentiva della vita i pesi./ Di mano ei gli sfuggi come un pallone.// "Non somigliare - ammoniva - a tuo padre"./ Ed io più tardi in me stesso lo intesi:/ Eran due razze in antica tenzone".

Apparentemente, il modello da assumere per il poeta è la madre (e la figura materna in generale, compresa la sua famosa balia slava Peppa); la figura paterna (un padre che Saba ha conosciuto solo ventenne), viene presentata inizialmente con il brutale aggettivo assassino. Eppure la sua figura gaia e leggera, in contrasto con quella della madre (tutti sentiva della vita $i$ pesi), mette in dubbio la sua reale posizione nel conflitto fra l'ascendenza paterna e materna, o fra le due razze. Un elemento di ambiguità questo che si può solo ipotizzare, ma che si giustifica con una grande integrazione degli ebrei italiani nella società italiana della fine del secolo scorso e dell'inizio di questo. A questo proposito è significativa la testimonianza dello storico italiano di origine ebraica Arnaldo Momigliano che (in Pagine ebraiche) afferma: "ciò che ... è più tipico degli Ebrei italiani è che durante il $\mathrm{XX}$ secolo essi sono arrivati ad avere un ruolo molto importante nell'amministrazione statale come funzionari, giudici, e sopratutto soldati" (p. 137). Nello stesso testo Momigliano mette in luce "l'importanza decisiva degli Ebrei di Trieste nel cosiddetto irredentismo, la rivendicazione di Trieste all'Italia" (p. 138).

Di Saba è già stato sottolineato il carattere cos̀ ebraico della sua produzione. Si veda questa affermazione di Ungaretti: "L'originalità di Saba è in quella sua potenza equivoca (direi oscena, ebreissima), senza intelligenza, e quindi non introspettiva, tutta, esclusivamente, sentimentale, sessuale: cieca"1 Occorre notare che Ungaretti rasenta in queste affermazioni una punta di critica razziale. M. Lavagetto, un critico che recentemente si è occupato molto di Saba, ha scritto: "II canzoniere è un 'romanzo' ebraico, la storia di un uomo la cui 'diversità' ... è amplificata da un destino prenatale" (ib., p. 36).

Nel panorama della poesia italiana del Novecento, la figura di Saba si è imposta all'attenzione fin dal 1928, quando gli è stato

1 UNGARETTI, G. "Lettera a E. Cecchi del 17.5.1926" In: SABA, U. La malinconia amorosa. Milano: Rizzoli, 1992, p. 31. 
dedicato un numero della rivista Solaria. Saba è il poeta del quotidiano, di una Italia delle piccole cose, che focalizza nei minimi movimenti un'attenzione e un'affettività estreme ("io ritrovo, passando, l'infinito/ nell'umiltà", da Città vecchia, e "Amai trite parole che non uno/ osava. M'incantò la rima fiore,/ amore...", da Mediterranee). Distanziandosi sia dalla scelta di stile roboante e carica di D'Annunzio, sia dalle sottili assonanze degli ermetici, al di qua della linea di violento sovversivismo linguistico del futurismo, Saba stesso, agli esordi nel 1911, definisce semplicemente onesto il suo linguaggio ("Ai poeti resta da fare la poesia onesta"2), quasi volesse interpretare nello pseudonimo da lui scelto un linguaggio che "dice pane al pane": una metafora delle sue scelte stilistiche. Di fatto, la scelta di Saba è legata alla trasparenza, alla trasmissione diretta della carica semantica nuda delle sue parole: "Parole,/ dove il cuore dell'uomo si specchiava/ nudo e sorpreso - alle origini..." (da Parole). Questa voluta ricerca di ciò che è scarno, povero - le trite parole - ricorda certamente i poeti crepuscolari (Gozzano, per esempio) e costituisce insieme un punto di forza e di debolezza della poesia di Saba, rinchiusa in una visione alquanto tradizionale della forma (si definiva un "poeta fedele alla tradizione"). Commenterà più tardi Edoardo Sanguineti che il suo 'conservatorismo' e, insieme, ostinato 'autobiografismo' ritardarono l'equa valutazione del suo lavoro di poeta, quando non ne favorirono, più tardi, improprie apologie"3

In Saba ebraismo e psicanalisi, ciascuno dei quali molto periferico nella cultura italiana, si toccano, come le tangenti ideali di due parallele, intersecazione di culture: quella mitteleuropea (di lingua e tradizione tedesca) e quella italiana, dato che la tradizione italiana a Trieste è miracolosamente sopravvissuta a secoli di dominazione austriaca. $\mathrm{O}$ forse meglio: è nata come il prodotto originale di un'incubazione austroungarica, dando a questa città un ruolo di cerniera e contatto fra culture molto diverse. Nella zona di Trieste al tempo romano esisteva un piccolissimo centro e così durante l'auge della gloriosa Repubblica di Venezia; solo a partire dall'istituzione del porto franco (secondo una decisione dell'imperatrice austriaca Maria Teresa) la minuscola cittadina si espande, per arrivare ai 220 mila abitanti dell'inizio del secolo. La sua caratteristica di avamposto europeo proiettato tramite l'Adriatico sul Mediterraneo ne farà il luogo ideale per l'incontro fra

2 LAVAGETTO, M. La gallina di Saba. p. 18

3 SANGUINETI, E. "U. Saba". In: Poesia italiana del Novecento. p. 781 
oriente e occidente, Europa settentrionale e meridionale, e permetterà a una consistente colonia ebraica di prolificare e, allo stesso tempo, la vicinanza rispetto a Vienna le permetterà, all'inizio del secolo, di diventare un centro di irradiazione della psicanalisi in Italia.

$\grave{E}$ una singolare coincidenze che Trieste, in fondo tanto periferica, abbia avuto due letterati ebrei della statura di Umberto Saba e Italo Svevo. Gli elementi in comune fra i due scrittori vanno ben al di là di questo mero dato anagrafico. Entrambi fanno dell'italianità una bandiera. Entrambi manifestano molto precocemente un interesse spiccato per la psicanalisi: Italo Svevo è certamente il primo scrittore a dedicare al tema della psicanalisi un libro (La coscienza di Zeno) in cui la nuova disciplina (la nuova visione del mondo?) entra come tema (uno dei capitoli si chiamerà "psico-analisi" ed il protagonista Zeno è affetto da una serie di sintomi nevrotici) e come pretesto letterario (la psicanalisi come strumento di interpretazione della realtà e come sostituto della critica letteraria). In Saba la psicanalisi viene riconosciuta fin dall'inizio come elemento ispiratore, al punto da meritare la brillante osservazione di Gianfranco Contini di un Saba "psicanalitico prima della psicanalisi"4 Non a caso il personaggio Zeno di Svevo, in polemica con i dottori dell'epoca, rivendica un primato sulla psicanalisi e sulle scoperte di Freud ("Giunsi a parlare con lui come s'egli avesse potuto intendere la psico-analisi ch'io, timidamente, precorsi") ${ }^{5}$ Forse anche nelle scelte stilistiche (dimessa, trasparente, essenziale la lingua di Saba e ostica, barbara, anacolutica quella di Svevo) c'è una affermazione quasi involontaria della diversità, manifestazione di una visione periferica e decentrata (rispetto alla tradizione italiana), pur ideologicamente respinta. "La psicoanalisi è una grande cosa - scrive Saba a Comisso nel 1929 - ...Essa può, dopo una lunga disciplina, portare alla coscienza dei fatti, o meglio, dei sentimenti rimossi; e dare quindi alla coscienza dell'uomo una maggiore estensione in profondità; se l'uomo è un artista può, di riflesso, risentirsene anche la sua arte"6. La coscienza di cui parla Saba è come il prodotto di una lettura posteriore della raccolta dei suoi testi e, principalmente, delle sue poesie. Così come, per il personaggio Zeno, la coscienza è il prodotto di una rilettura a posteriori, una mimesi autobiografica. Un modo come un altro per testimoniare l'ingresso

4 LAVAGETTO. Op. cit., p. 15

5 SVEVO, Italo. La Coscienza di Zeno. Roma: Newton Compton, p. 396

6 Apud: LAVAGETTO. Op. cit, p.16. 
delle tematiche psicanalitiche nella letteratura contemporanea. Dirà scherzosamente Carlo Emilio Gadda (che di psicanalisi se ne intendeva) "Appare dai poetici testi ch'egli (Saba) abbia trasferito, ch'egli abbia concentrato sulla sua balia, la Peppa, tutta la intensità iniziale (la libido, se permettete) del suo animo, della sua psiche di fanciullo... Quel caffelatte, sostengono gli psicanalisti, non è che un succedaneo, un Ersatz (ma loro dicono un transfer [sic]) delle erogazioni naturali della Peppa"7 Ė da notare che sia Saba che Svevo adottano degli pseudonimi: se $S a b a$ abbandona il cognome paterno, Svevo abbandonerà il cognome originale Schmitz (di origine ebraica e ungherese), un fatto che viene interpretato dallo stesso autore in maniera varia: Svevo, da Schwabe un omaggio alla Svevia o a Schopenhauer che, comunque, rispetto al nostranissimo Italo denota una divaricazione, un contrasto. Ancora un caso vuole che un banalissimo caffellatte (citato da Gadda ironicamente) svolge un ruolo di primissimo piano nella conclusione dell'ultimo romanzo di Svevo, laddove il protagonista rimpiange più della perdita del contatto con la sua famiglia, la rinuncia forzata della prima colazione allo scoppio della prima guerra mondiale. Il tema della psicanalisi, nei due autori triestini di origine ebraica, appare come il prodotto di una maggiore libertà dalle tematiche più "impegnate" che si affermavano nel resto del panorama della cultura italiana, sempre alle prese con un risvolto politico, ideologico, del resto imposto agli intellettuali dalla realtà del regime fascista.

La psicanalisi, oltre al problema della lingua (fra un dialetto triestino, il tedesco e il toscano, definizione della lingua italiana in $\mathrm{La}$ coscienza di Zeno), potrebbe rappresentare questo ritorno del rimosso nell'attività letteraria dei due scrittori, evidenza della diversità, che dirotta la ricerca letteraria verso i meandri dell'io contemporaneo, in cerca di una risposta sulla questione delle origini (in indiretta polemica con i temi ideologico-culturali interessanti il futurismo o le roboanti affermazioni di certa letteratura decadentista). In Saba il problema delle origini si evidenzia nel contrasto (presunto o reale) fra origine e ascendenze paterne e materne, dove - come si sa e come risulta dall'acquisizione del suo nuovo cognome di origine ebraica - le sue preferenze sono chiare a favore dell'ascendenza ebraica.

Le "due razze in antica tenzone" citate nell' Autobiografia n. 3 - in realtà qui più una mascheratura del rapporto fra un padre

7 GADDA, C. E. "Letteratura e psicanalisi". In: I viaggi e la morte Milano: Garzanti, 1987. 
assente e la forte figura materna che un contrasto fra ebraismo e "arianesimo"- avranno il loro concreto riflesso quando, anni più tardi, il regime fascista promulgherà le famigerate leggi sulla razza, tramite le quali gli ebrei verranno perseguiti, impedendo loro qualsiasi attività economica ufficiale. Giuseppe Ungaretti ricorderà in seguito di essere intervenuto a favore del poeta in un breve soggiorno in Italia, durante il periodo della sua residenza in Brasile: "Erano entrate in vigore da noi le leggi razziali, e Saba, che non avrebbe più potuto gestire la sua bottega di libri antichi, e che temeva per la sua famiglia e per sé anche più gravi persecuzioni, era partito fuori di sé per Parigi [e] minacciava di uccidersi..." . L'intervento di Ungaretti riuscirà nell'intento anche se Saba finirà per rifugiarsi prima a Parigi e poi, clandestino, a Firenze, per evitare i rastrellamenti.

"Il ghetto di Trieste nel 1860" fa parte di un ciclo di cinque racconti (Gli ebrei) che Saba aveva intenzione di raccogliere insieme ad altri in un volume e che apparirà solamente in una edizione del 1956. I motivi di questa dilazione non sono noti: oltre al rifiuto della rivista La Voce a pubblicare l'insieme dei racconti devono aver pesato alcune rimostranze e critiche rivolte all'autore dalla famiglia, che vedeva forse traditi ricordi gelosamente custoditi nella memoria familiare (in particolare le critiche gli furono rivolte dalla zia Regina, a cui i testi sono dedicati). Forse, però, a ritardarne la pubblicazione è stato un tono ironico, al punto di arrivare al sarcasmo e all'autolesionismo. Dirà Saba nella prefazione alla raccolta (scritta nel 1952): "I cinque racconti... furono scritti quando l'antisemitismo pareva un gioco: ed io potevo, senza rimorso, abbandonarmi alla comprensiva ironia, venata di nascosta tenerezza, verso persone e cose ... che conobbi e vidi, o di cui, più spesso, ho sentito parlare, al tempo della mia fanciullezza. Mia madre - come si sa - era ebrea, ed ebrea era tutta la sua famiglia... L'esito infelice che ebbe il matrimonio di mia madre, l'abbandono pochi mesi prima che io nascessi, l'aveva rinforzata nei suoi pregiudizi religiosi e razziali...". In fondo, però, l'ironia estrema del testo mostra un Saba molto più nostalgico verso perdute storie familiari che realmente interessato nel difendere dei pregiudizi razziali (per di più adottando il punto di vista ebraico, di una madre polemica contro l'altro sesso e "l' altra razza"). Si tratta di pagine di puro folklore, elementi del colore locale che, nella loro crudezza, rispecchiano an-

8 UNGARETTI, G. "Peregrinazione con Umberto Saba" (del 1957, in occasione della sua morte) In: Saggi, Milano: Mondadori, 1982, p. 675

9 LAVAGETTO, M. Per conoscere Saba. Milano: Mondadori, 1981, p. 85-6. 
cora una volta la vocazione di Saba verso una sincerità e una schiettezza, al di la di qualsiasi ideologia. A meno che non vengano interpretate come pagine del "ritorno del rimosso", che portano alla luce un peso maggiore del mio sguardo azzurrino, che Umberto Poli avrebbe voluto negare.

\section{II \\ IL GHETTO DI TRIESTE NEL 1860}

Verso il 1860 il Ghetto di Trieste era ancora in piena fioritura della sua sudicia originalità. Da mezzo secolo uguagliati agli altri cittadini, liberi da particolari gabelle e distintivi umilianti, non tutti gli ebrei nati od emigrati nella città portofranco, avevano appreso a vincere l'ingenita diffidenza a mescolare la loro vita quotidiana a quella dei temuti (e quindi odiati) "goim". Quest'avversione, che non era religiosa, e che, dove esista, il battesimo non cancella, radicata com' è nella stirpe da millenni di persecuzioni e quarentene, tratteneva anche famiglie sufficientemente agiate per abitare una casa nuova in una via nuova, nella "cittadella" dove i loro vecchi avevano esercitato ed esercitavano tuttavia il commercio delle robe usate, nelle cui botteghe pittoresche e caotiche stava la radice della loro forza. Le case nuove, costruite come eccellente impiego di capitali per ricche vedove e timorosi di rischi maggiori, erano, è vero, il sogno di molti; ma, acquistate per speculazione, i nuovi proprietari continuavano, per conto proprio, ad abitare in quel ben amato Ghetto, pieno per essi di intimità e memorie. Per virtù della tradizione e la forza d'inerzia di un'abitudine mentale diventata, come una qualunque idea fissa, un peso più difficile ad essere deposto che portato, s'immaginava volentieri che, fuori di quel centro, durassero ancora persecuzioni cessate da decine d'anni, e che non erano mai esistite nell'unico grande porto mercantile dell'Austria, dove la popolazione aveva un carattere già troppo meridionale perché la malattia nordica dell'antisemitismo vi potesse attecchire. Fu così che, schiuse appena le porte del Ghetto, i migliori ebrei, quelli liberi dal pregiudizio e dalla paura, seppero, con la potenza del denaro accumulato, $o$ in virtù della praticità propria alla razza che ha forse più idealismo nel commercio $e$ più commercio nell'idealismo, collocarsi d'un balzo nei seggi più autorevoli $\mathrm{e}$ lucrosi; alla direzione delle Banche, delle Compagnie di Assicurazione e di Navigazione. Era il tempo in cui la Comunità si accresceva ogni giomo di nuovi correligionari, attratti dalla crescente floridezza dei traffici, che andavano mutando 
a vista d'occhio la vecchia cittadina di pescatori in un'enorme tumultuante bottega. E molti che, dal vicino Levante, sbarcavano al Molo San Carlo con la giubba a brandelli e il fez rosso in testa, senz'altra ricchezza che, forse, una commendatizia per il Rabbino o qualche filantropico vecchio, si vedevano, dopo pochi anni, a volte mesi, comparire in abito di rigore e cappello a cilindro alle solennità religiose, nei tre tempi, di rito italiano, tedesco e spagnolo; due dei quali si aprivano ai fedeli nel Ghetto propriamente detto, ed il terzo non lontano: in Via del Monte. Ma il popolo minuto, quelli che non avevano abbastanza iniziativa per liberarsi dal piccolo commercio, continuavano a esercitarlo nelle baracche della Piazzetta detta delle Scuole Israelitiche, o al pianterreno delle case umide e dei postriboli di città vecchia. Alcuni di questi negozi, grandi come celle e produttori di capitali ingenti, rimasero leggendari nella nuova generazione. Vi si dibatteva, più d'ogni altro, il baratto e la compravendita dei mobili e degli indumenti usati, industria oggi quasi scomparsa, i cui ultimi grandi attori sono più vecchi e più rari che $i$ pochi autentici superstiti dell'epopea garibaldina. I clienti erano in gran parte slavi del Territorio e marinai dell'llliria e della Dalmazia: coscritti che avevano bisogno di una valigia in cui deporre il loro misero corredo; marittimi che volevano scanmbiare il loro vestito logoro e lo loro scarpe scalcagnate con un vestito un po' meno vecchio e scarpe un po' meno scalcagnate. I furti, gli imbrogli, le usure (non più frequenti, né più gravi che altrove, ma più scoperti, più - per usare un termine topicamente improprio - "alla luce del sole") erano esercitati dai mercanti, e più dalle mercantesse, con un'abilità addirittura sublime, con una stupefacente conoscenza psicologica e fisiologica delle vittime. Tutte le armi erano buone; sia per attirare nel proprio negozio il cliente, sia per vincere la concorrenza con gli altri rivenditori. Le vie erano strette, le androne affollate, e le botteghe aperte, una di fronte all'altra, come campioni nemici, al tempo che le battaglie si vincevano o si perdevano con combattimenti corpo a corpo. I passanti sospetti compratori erano assaliti con le più sfacciate lusinghe, come i nottambuli dalle prostitute. Se era un giovanotto lo si adescava con parole di lode alla sua robustezza, parole sfuggite sicuramente ad un disinteressato entusiasmo; e la venditrice gli faceva comprendere che gli avrebbe infilato con le sue stesse mani il nuovo indistruttibile paio de brache, e che, per preferenza a un così bel biondo, gliele avrebbe lasciate quasi gratis. Ma quel "quasi" era, a volte, la giornata di una famiglia, con otto figlioli da sfamare, e nessuno, caduto una volta nella trappola, ne usciva, un'ora o minuto dopo, senza averne fatte, almeno in parte, le spese. Se, invece di un giovanotto, era un pade di famiglia, si accarezzava il marmocchio che gli sgambettava dietro, lo si proclamava il più bel maschietto della città; ciascuno se n'augurava, ad alta voce, uno simile; si mutava lingua, dialetto, sorriso, secondo l'età, il sesso, la nazionalità del cliente. Per i casi gravi, quando l'affare, lungamente discusso, minacciava di non arrivare in porto, c'era, nascosta dietro il banco come 
estrema riserva, una bottiglia d'acquavite, con uno o due sudici bicchierini. Fra padrone e padrona, fra madre e figlia, si parlavano, durante la compravendita, un gergo composto di un triestino dolciastro, inframmezzato di vocaboli del basso ebraico, incomprensibile ai non iniziati. Più accaniti ancora erano nella concorrenza fra di loro; i compratori se li disputavano fin con la forza: non era raro il caso che di due, o più concorrenti, vincesse quello che aveva una muscolatura meno debole alle braccia, e quindi una maggiore capacità a trascinare nella sua bottega il "goi", lusingato o divertito da quest'insolita lotta intorno alla sua persona. Allora la parte soccombente sfogava il suo rancore, urlando dalla soglia del suo negozio tutti i segreti commerciali e tutte le turpitudini domestiche della parte avversaria. O passava, tutt'a un tratto, alle querele, numerava le sue miserie, il numero di bocche che doveva sfamare, con una loica barbarica, con sprazzi d'eloquenza degni di Geremia. Era insomma una lotta per la vita cos̀ furiosa quale si ammira negli insetti tra l'erbe del prato o la rena del mare. Ma i padroni di botteghe già avviate, e con una clientela ormai stabile, si prendevano la religiosa soddisfazione di tenerle chiuse il giorno di Sabato, il giorno del Signore, fino al cui tramonto è vietato all'ebreo ogniqualunque lavoro, anche strappare una foglia, anche accendere un fiammifero. lo stesso ho udito dei vecchi raccontare con orgoglio e commozione che una vera folla si accalcava il Sabato sera alle porte di quelle botteghe, attendendo per ore il padrone o la padrona che le riaprisse. Perché - aggiungevano - quelle botteghe erano benedette (da Dio); erano una vera terra promessa.

NOTA

Goi: non "iudi"; non ebreo.

Abstract: The poet of Trieste, Umberto Saba, in the light of his Jewish roots and psychoanalysis that distinguish his biography and it is the key of lecture of his poetical production.

Keywords: Umberto Saba, contemporary Italian poetry, Italian literature, psychoanalysis. 